\title{
Estimación de la vulnerabilidad del acuífero del valle de Toluca mediante la combinación de un método paramétrico y el transporte advectivo
}

\author{
J. Gárfias(1), H. Llanos ${ }^{(2)}$, R. Franco(1) y R. Martel(3) \\ (1) Universidad Autónoma del Estado de México. Facultad de Ingeniería (CIRA). 50130Toluca, México. \\ jgarfiass@gmail.mx \\ rfranco@uaemex.mx \\ (2) Universidad del País Vasco. Departamento Geodinámica. 1006 Vitoria-Gasteiz, País Vasco. \\ gppllach@ehu.es \\ (3) Institut National de la Recherche Scientifique. Centre Éau, Terre \& Environnement, \\ 490 rue de la Couronne, Québec (Qc), G1K 9A9, Canada. \\ richard.martel@ete.inrs.ca
}

\begin{abstract}
RESUMEN
La estimación de la vulnerabilidad del agua subterránea es una tarea importante en la administración de los recursos hídricos, que depende de la disponibilidad de datos y complejidad de las condiciones hidrogeológicas. Este estudio, que integra el uso de un enfoque combinado basado en un método de vulnerabilidad (DRASTIC) y el transporte advectivo, permite comprender mejor la susceptibilidad a la contaminación en el acuífero de Toluca. Para estimar el transporte advectivo y el flujo regional del agua subterránea se ha desarrollado un modelo en 3D usando VisualModflow y MODPATH. El mapa de vulnerabilidad demuestra que la aplicación e interpretación del método paramétrico es problemática, presentando una diferencia aproximada del $23 \%$ si éste es comparado con el mapa modificado de vulnerabilidad. La vulnerabilidad a la contaminación de los vertederos de residuos sólidos es relativamente alta; aproximadamente el $76 \%$ están localizados en áreas que pueden ser susceptibles a la contaminación a través de la infiltración vertical, especialmente aquellos que están ubicados a lo largo del sistema Lerma. Los parques industriales, donde ocurre una extracción continua de agua subterránea y subsidencia del terreno, han sido clasificados como zonas de vulnerabilidad alta, incrementando el riesgo de contaminación de fuentes superficiales que podrían alcanzar el agua subterránea. Con el objeto de entender la susceptibilidad a la contaminación en el acuífero, varios enfoques de vulnerabilidad deben ser adoptados y todos los resultados que permitan una validación entre ellos deben ser considerados, constituyendo una buena estrategia en la definición de diferentes niveles y medidas de protección.
\end{abstract}

Palabras clave: Vulnerabilidad, protección del agua subterránea, modelación numérica, DRASTIC, valle de Toluca

\section{Vulnerability assessment of the Toluca Valley aquifer combining a parametric approach and advective transport}

\begin{abstract}
Groundwater vulnerability assessment is an important task in water resources and land management. Depending on the availability of data and the complexity of the hydrogeological conditions, different approaches can be adopted. As an alternative, this study involves the use of a combined approach based on vulnerability methods and advective particle tracking to better understand the susceptibility to contamination in the Toluca valley aquifer. An intrinsic vulnerability map (DRASTIC) was used to identify areas that are more susceptible to ground water contamination. To estimate advective particle tracking, we developed a 3D flow model using VisualModflow and MODPATH to describe the regional flow of groundwater. The vulnerability map demonstrates the problematic application and interpretation of qualitative the vulnerability method
\end{abstract}


Gárfias, J., et al., 2017. Estimación de la vulnerabilidad del acuífero del valle de... Boletín Geológico y Minero, 128 (1): $25-42$

of the parametric system group, which indicates a difference of approximately $23 \%$ when compared with the modified vulnerability map. Potential contamination sources based on landfill sites were comparatively high; approximately $76 \%$ are located in areas that could be susceptible to contamination through vertical infiltration, especially those that are located along the Lerma system of wells. Industrial parks located in the centre of the valley (83\%), where continuous extraction of groundwater and land subsidence occurs, have been classified as high vulnerability zones, increasing the risk of contaminants from surface sources reaching the groundwater. In order to understand the susceptibility to contamination in the aquifer, various delineation approaches should be adopted and all the results that validate each other should be considered, thus making a good strategy for implementing different degrees of protection measures.

Keywords: vulnerability, groundwater protection, numerical modelling, DRASTIC, Toluca valley

\section{ABRIDGED ENGLISH VERSION}

\section{Introduction}

Vulnerability maps illustrate the potential threat of contamination to groundwater and can be considered as important tools for land-use planning and related legislation. For volcanic aquifers the estimation of the susceptibility to contamination is important, especially in regions where groundwater from these aquifers is the primary source of water for municipal, agricultural and industrial uses. However, the resulting qualitative maps are often inconsistent and even contradictory and thus might lead to inconclusive vulnerability assessments. In order to improve this situation, this study involves the use of a combined approach based on vulnerability methods and advective particle tracking to better understand the susceptibility to contamination in the Toluca valley aquifer, where extensive pumping, agricultural, and industrial activities are concentrated (Figs. 1 and 2). The multidisciplinary approach not only demonstrates the utility of groundwater contamination risk mapping of the overexploited aquifer, but also provides excellent tools for land use and the protection of water resources.

\section{Methods and results}

The assessment of groundwater contamination risk requires two main factors: establishing the conceptual and operational basis for combining vulnerability methods and identifying potential sources of pollutants and their possible impact. In this study, the modified vulnerability map was coupled with land use to represent susceptibility to contamination. The impact map was based on two factors: the impact of major potential contaminants in the area of study and the water supply capture zone of the wellfields. During this research, the DRASTIC intrinsic vulnerability map was used (Hamza et al., 2015). DRASTIC is based on seven hydrogeological parameters: depth to water table, recharge, aquifer media, soil media, topography, influence of the vadose zone, and hydraulic conductivity to assess the intrinsic aquifer vulnerability. Each map was classified and rated, then weighted based on the standard DRASTIC weight system (Aller et al., 1985). The vulnerability index is the sum of each rated map multiplied by its respective weight. The final DRASTIC product is a map showing the vulnerability index. Once the DRASTIC indices had been computed, it was possible to identify areas that are more susceptible to ground water contamination than others (Fig. 4). The higher the DRASTIC index is, the greater the potential for groundwater contamination. The DRASTIC index only provides a relative evaluation tool and is not designed to provide absolute answers. To estimate the advective particle tracking, we developed a 3D flow model using the MODFLOW software and the MODPATH semi-analytical algorithm to describe the regional flow of groundwater in the aquifer within the basin. The simulated aquifer was divided into two layers with different hydraulic conductivities based on borehole descriptions. The upper layer represents the semi-confined and unconfined aquifer containing heterogeneous alluvial sediments and the lower layer represents the semi-confined aquifer and consists primarily of fractured volcanic material (Hancox et al., 2010; Calderhead et al., 2011). Figure 3 outlines the three-dimensional conceptual scheme of the Toluca Valley aquifer and the boundary of the groundwater model. The finite difference grid has 94 rows and 82 columns (Fig. 4), and each block is 500 $\mathrm{m}$ by $500 \mathrm{~m}$. General head and drain boundaries were used as boundary conditions and a value of $55 \mathrm{~mm} /$ year was assumed as average recharge. The model used to simulate the average conditions was calibrated to 1970 steady-state and 1970-1996 transient conditions. To estimate the impact of potential sources, 1996 was chosen as the steady-state condition to determine advective travel times. Once the groundwater flow was calibrated, advective particles were inserted around the wellfields and tracked in the upgradient 
direction for advective travel times for 50 years. Time-of-travel zones, as well as steady-state capture zones, have been determined in this way. Figure $6 \mathrm{~b}$ shows the particle tracks for the Toluca valley aquifer wellfields as horizontal plane projections at 50 years. Figures 4 and 6a show that areas close to the Nevado de Toluca and the southeast area around the Sierra de La Iglesia have the highest vulnerability. This is because the lithologic stratigraphy of the units has high permeability in this area and the area is covered by fractured volcanic formations. In addition, this area receives the highest recharge rates. On the contrary, the central area and the northeast region of the Toluca valley has the lowest vulnerability because the recharge rates are low and the superficial geology consists primarily of volcanic material associated with reduced infiltration rates. Figure 4 displays the spatial distribution of the Toluca valley aquifer factors and the groundwater vulnerability map generated according to the DRASTIC method. Due to the qualitative nature of the parametric vulnerability maps, the parametric approach was modified to include advective particle tracking to determine flow paths and advective travel times for the wellfields. The contamination risk was quantified by analyzing the impact of potential sources of pollutants located within the capture zone at the wellfields. Figure 5 displays the discretisation of the study area and the boundary conditions of the groundwater model and the comparison of simulated and observed groundwater levels under steady-state calibration for 1996. In this context, the modified index vulnerability map changed from $68 \%$ to $50 \%$ for low vulnerability and from $21 \%$ to $16 \%$ for medium vulnerability, whereas the high vulnerability areas were modified from $11 \%$ to $34 \%$. Analysis of the results revealed that the risk of well-field contamination and the possible impact from landfill sites were comparatively high. Similarly, the potential contamination from industrial zones is relatively high, especially in the centre of the valley where continuous extraction of groundwater and land subsidence occurs. Figures $6 b$ and $6 c$ summarize the results from particle tracking flow paths from the well-fields for 50 years of the capture areas (4 scenarios) and the combined groundwater vulnerability map derived from DRASTIC and numerical modelling approaches, including industrial zones and landfills sites.

\section{Discussion and conclusions}

Vulnerability maps illustrate the potential threat of contaminants to groundwater and can be considered as important tools for land-use planning and related legislation. For volcanic aquifers, where densely populated urban communities and industrial parks are located, vulnerability maps are excellent tools for source and resource protection. However, the resulting qualitative maps are often inconsistent and even contradictive and thus might lead to inconclusive vulnerability assessments. Results show that the classic DRASTIC approach may underestimate groundwater vulnerability in settings such as the one under study. The proposed approach for the delineation of groundwater protection areas in the study area, based on vulnerability methods and advective particle tracking, effectively complements the complex hydrogeological behaviour of the volcanic aquifer system. The vulnerability map of the test area demonstrates the problematic application and interpretation of the qualitative vulnerability method of the parametric system group, which indicates a difference of approximately $23 \%$ when compared with the modified vulnerability map. The potential contamination sources based on landfill sites were comparatively high; approximately $76 \%$ are located in areas that could be susceptible to contamination through vertical infiltration, especially those that are locate along the Lerma system of wells. Additionally, industrial parks located in the centre of the valley (83\%), where continuous extraction of groundwater and land subsidence occurs, have been classified as high vulnerability zones, increasing the risk of contaminants from surface sources reaching the groundwater. To sum up, in order to understand the susceptibility to contamination in the Toluca Valley aquifer, various delineation approaches should be adopted and all the results that validate each other must be considered, thus providing a good choice in implementing different degrees of protection measures.

\section{Introducción}

En la actualidad es muy común la alteración de las características naturales de las aguas, ya sean superficiales o subterráneas, lo que pone en riesgo muchas de las actividades humanas y trae consigo una pérdida de la calidad de vida, incluyendo peligros sanitarios importantes. En particular, las aguas subterráneas son más difíciles de contaminar que las superficiales, pero cuando se produce es muy persistente y difícil de eliminar, siendo la purificación técnicamente problemática, excesivamente costosa y con frecuencia poco práctica, debido a que tienen un ritmo de renovación muy lento. A este respecto, la situación de México no es nada halagüeña, enfrentándose a graves problemas, entre los que destacan la ausencia de herramientas de protección frente a la contaminación y la disminución acelerada de la 
disponibilidad de agua (Rudolph et al., 2006; Gárfias et al., 2008a; CONAGUA, 2014).

Esta disminución comenzó con el crecimiento de la ciudad de México localizada a $60 \mathrm{~km}$ de la cuenca del valle de Toluca, cuyos recursos han constituido una importante fuente de agua potable, inicialmente captando sus principales manantiales $y$, desde 1951, con la entrada en operación del sistema Lerma constituido por una batería de 236 pozos de los que se extrae un caudal instantáneo de $6 \mathrm{~m}^{3} / \mathrm{s}$ con destino al Distrito Federal (Fig. 1) (Hancox et al., 2010; Calderhead et al., 2012a). Así mismo, dentro de la cuenca existe una demanda significativa de agua para usos agrícolas e industriales, sin olvidar las necesidades de abastecimiento en la ciudad de Toluca y de los municipios del valle dependientes exclusivamente de los recursos subterráneos. Esta situación ha llevado a la sobreexplotación del agua subterránea, ocasionando el descenso de los niveles piezométricos $(60$ cm/año), el cambio en las pautas del flujo subterráneo y la disminución progresiva de los cuerpos de aguas superficiales y zonas lacustres existentes en el Sur de la cuenca (Calderhead et al., 2012b; Expósito et al., 2010; Martín del Campo et al., 2014).

En este sentido, dada la importancia que presenta el estudio de la protección de las aguas subterráneas para el ordenamiento territorial, en general, y para el uso sostenible de los recursos hídricos, en particular, el objetivo principal del presente trabajo es evaluar la vulnerabilidad del acuífero del valle de Toluca, mediante la aplicación combinada de uno de los métodos de análisis más usuales, junto a la modelación numérica de flujo de agua subterránea con rastreo de partículas. Ello ha permitido elaborar un mapa de vulnerabilidad a la contaminación que sirva a las autoridades para la toma de decisiones en el planeamiento urbano e industrial, que garanticen, así mismo, la protección de la cuenca y de sus recursos naturales.

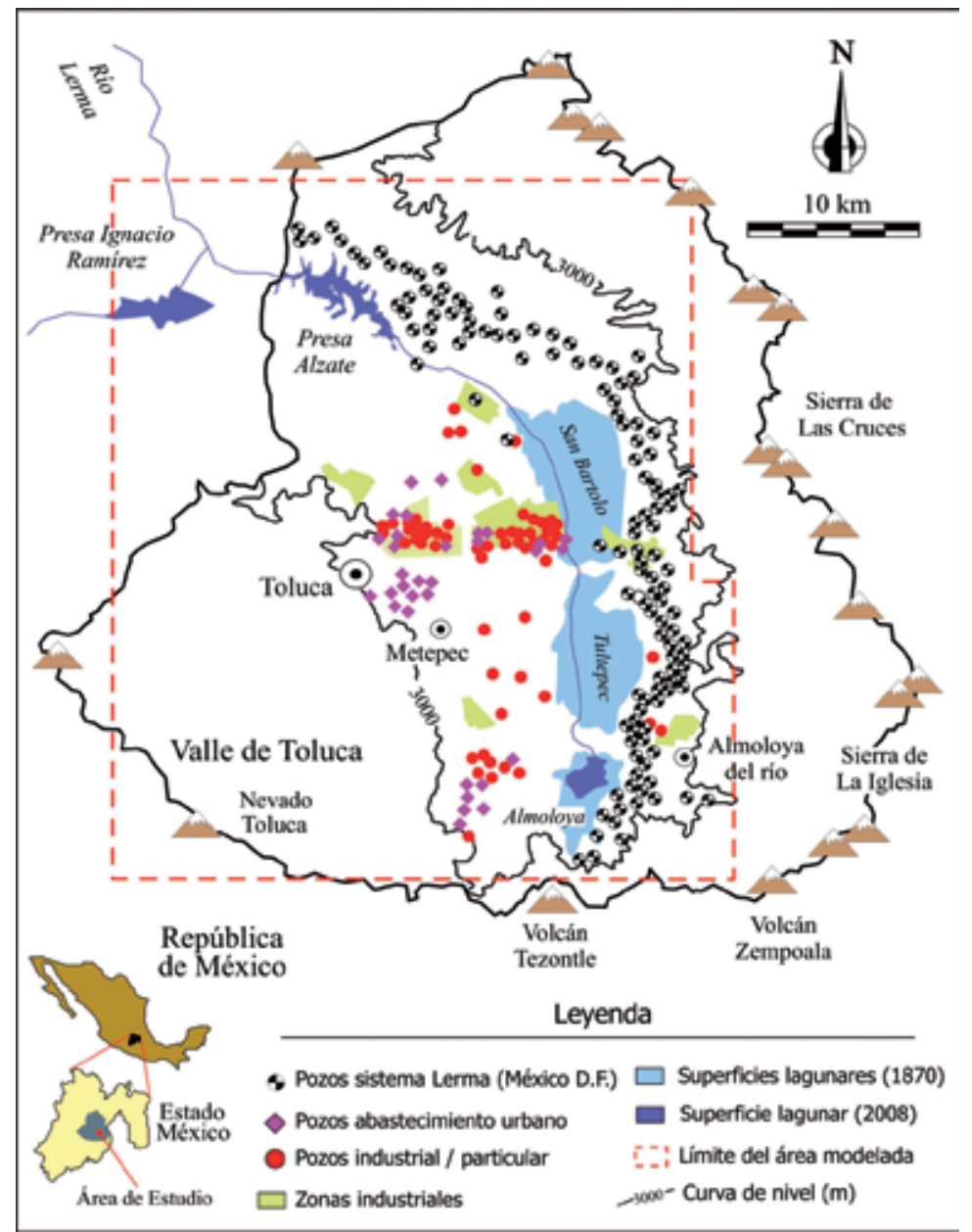

Figura 1. Mapa de localización del área de estudio que muestra las principales características fisiográficas, las zonas industriales, los pozos del sistema Lerma y los pozos de bombeo instalados en el valle de Toluca.

Figure 1. Location map of the study area showing the principal physiographic features, industrial zones, the Lerma system of wells and pumping wells installed in the Toluca valley. 


\section{Descripción de la zona de estudio}

La zona de estudio, localizada en el sector central del Estado de México, está constituida por un extenso valle de $2116 \mathrm{~km}^{2}$ y elevación media de 2600 msnm., rodeado, entre otras, por las elevaciones de las sierras de Las Cruces (4002 msnm) y del Nevado de Toluca (4680 msnm), que determinan la divisoria hidrográfica principal. Presenta un clima templado y subhúmedo, caracterizado principalmente con lluvias de mayo a octubre, y una precipitación media anual cercana a los $800 \mathrm{~mm}$ en el fondo del valle de Toluca, que se incrementa localmente hasta los 1300 $\mathrm{mm}$ en las faldas del Nevado, parte de la cual tiene lugar en forma de nieve.

El río Lerma recorre la cuenca de Sur a Norte y sus afluentes, cuyo nacimiento inicialmente se originaba en los manantiales situados al pie del volcán Tezontle en la región de Tenango del Valle dando origen a las lagunas de Almoloya del Río, Tultepec y San Bartolo (Fig. 1). Sin embargo, con el paso de los años, y de la mano de una excesiva explotación de los acuíferos, tanto los manantiales como, prácticamente, las lagunas han desaparecido (UAEM, 1993; Rudolph et al., 2006). El cauce del río Lerma es el colector que recibe y transporta hasta el embalse de Alzate las aportaciones de las aguas residuales de la ciudad de Toluca y poblaciones vecinas, así como del corredor industrial Toluca-Lerma, constituyendo actualmente una de las cuencas más contaminadas del país.

\section{Características Geológicas}

Desde el punto de vista geológico se inscribe en la llamada Provincia Fisiográfica del Eje Neovolcánico (Alaniz-Alvarez y Nieto-Samaniego, 2005; Ferrari et al., 2012), caracterizada por una intensa actividad volcánica que se manifiesta por la presencia de numerosos complejos volcánicos, conos cineríticos y domos andesíticos, que dieron origen, entre otros volcanes, al Popocatépetl, Iztaccíhuatl y Nevado de Toluca (García-Palomo et al., 2000), a cuyos pies se asienta la ciudad de Toluca, capital del Estado de México. Dicha actividad se inició durante el Terciario con un volcanismo generalizado de composición ácida a intermedia, que contribuyó a la formación de una serie de cuencas endorreicas en donde se depositaron gran cantidad de materiales granulares $y$ piroclásticos. De hecho el valle de Toluca, que actualmente está representado por una planicie en parte colmatada por materiales lacustres, constituye una de las antiguas cuencas rellenas por materiales piroclásticos y niveles vulcanoclásticos junto a depósitos aluviales derivados de la dinámica fluvial del río Lerma (García-Palomo et al., 2008).

La descripción y la simbología de las unidades litológicas, basadas en el estudio realizado por Ariel Consultores (Ariel Consultores, 1996), así como los trabajos desarrollados en la región del volcán de Toluca y áreas aledañas (García-Palomo et al., 2002), han permitido definir el esquema hidrogeológico general y la secuencia de unidades litológicas que se muestra en la Figura 2. Hidrogeológicamente los materiales se integran en dos medios desigualmente permeables: por porosidad básicamente intergranular y por porosidad debida a la fracturación (Fig. 2). El primero está representado por los depósitos clásticos que rellenan la cuenca del valle de Toluca. Incluye las siguientes unidades litológicas: tobas (Qtb) y arenas (Qtba), depósitos aluviales (Qal) y lacustres (Qla), los niveles de la formación Tarango (Tpt), además de los depósitos asociados al Nevado de Toluca (Qn y Qtbn), así como los conos volcánicos recientes de la formación Chichinautzin (Ob). A su vez, el medio fracturado integra las unidades de andesitas (Tpv y Qa), depósitos de la formación Las Cruces (Tomc) y andesitas de Xochitepec (Tomv), que a pesar de ser consideradas, a priori, como acuífugos o, en el mejor de los casos, como de baja permeabilidad, se encuentran afectadas por una intensa deformación reciente, lo que les confiere una porosidad secundaria, con una capacidad de albergar y transmitir agua localmente significativa. Esta característica se hace más evidente, si cabe, en los afloramientos cercanos a la divisoria de la cuenca, representando zonas de recarga importantes de los niveles acuíferos del fondo del valle con los que tienen continuidad hidráulica en profundidad.

\section{Hidrogeología y explotación del Acuífero}

El diagrama del modelo conceptual del acuífero (Fig. 3) muestra que el sistema está constituido por un primer conjunto granular de permeabilidad variable dispuesto sobre el medio fracturado de baja permeabilidad, en el que la recarga procede de la precipitación que tiene lugar en toda la cuenca. Una parte de ella se infiltra a través de las unidades desigualmente transmisivas localizadas hacia la divisoria hidrográfica principal, y otra parte se infiltra en la propia planicie que conforma el fondo del valle. En particular, los materiales de las formaciones Tarango (Tpt) y Cichinautzin ( $\mathrm{Qb}$ ) propician la infiltración de abundante precipitación, manifestada hacia el fondo de valle bajo la forma de los manantiales que desde tiempo inmemorial dieron lugar al sistema de 


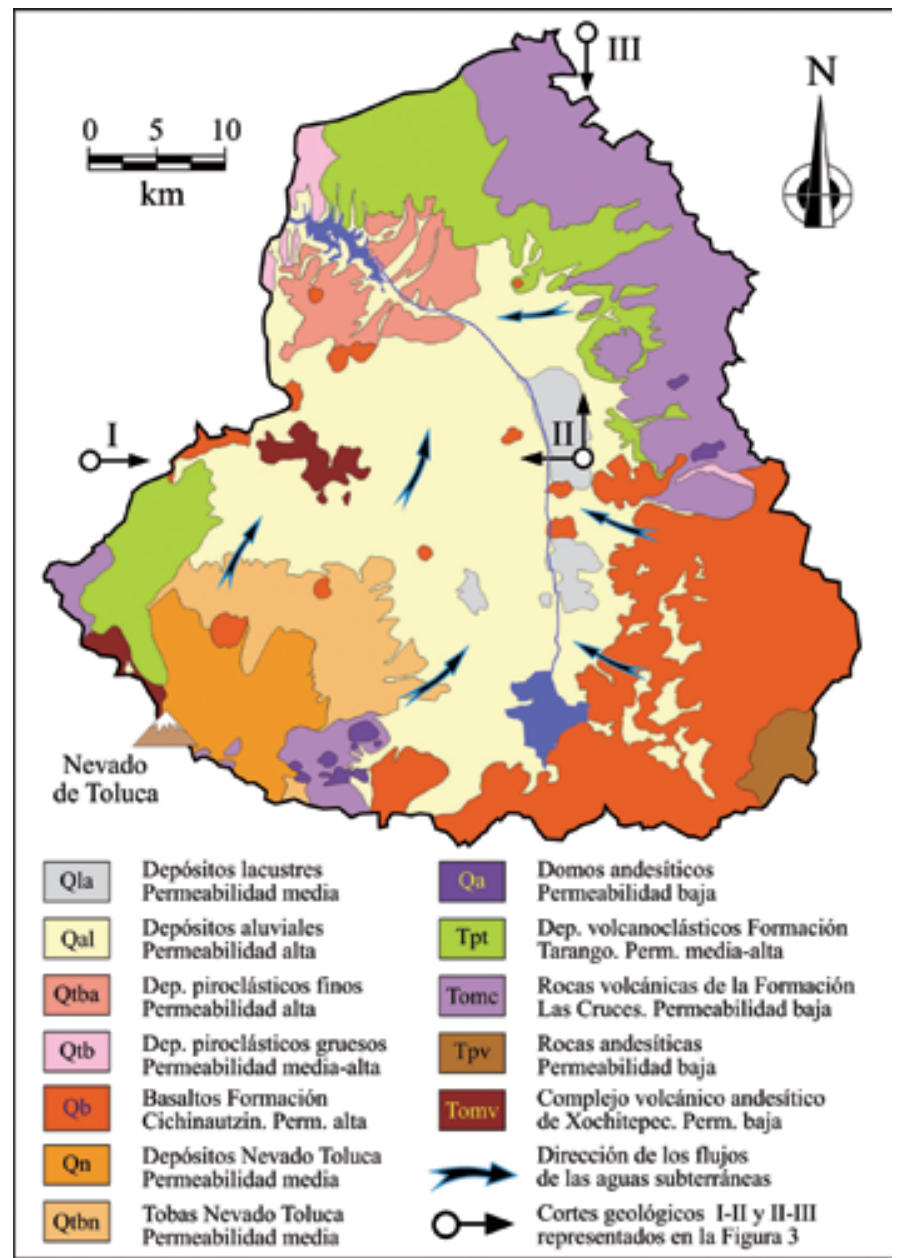

Figura 2. Esquema general hidrogeológico del acuífero del valle deToluca. Las flechas representan la dirección del flujo de agua subterránea. Figure 2. General hydrogeological scheme of the Toluca valley aquifer. The arrows represent the direction of the groundwater flow.

lagunas de Almoloya del Río (Figs. 1 y 3). Otro tanto sucede con los depósitos del Nevado de Toluca que proporcionan una importante recarga de tipo lateral (Gárfias et al., 2008a).

Así mismo, en el fondo del valle, constituido básicamente por materiales aluviales y tobas, se produce una elevada recarga con cargo a la precipitación directa $y$, en parte, por los numerosos cursos superficiales ocasionalmente influentes debido al descenso de niveles por sobreexplotación y que, por lo mismo, representan un riesgo de contaminación para el acuífero subyacente. Del mismo modo, se puede considerar una pequeña recarga por excedentes de riego. Por su parte, la descarga tiene lugar por los numerosos pozos de extracción existentes en la cuenca $y$, de forma natural, en dirección Norte hacia el vaIle de Ixtlahuaca a través del estrechamiento topográfico de Perales, coincidente con el cauce del río Lerma, el cual constituye la salida natural del sistema acuífero y de la cuenca (Fig. 3) (Hancox et al., 2010; Calderhead et al., 2012a).

\section{Análisis de la vulnerabilidad en la protección de acuíferos}

Los primeros mapas de vulnerabilidad desarrollados con carácter extensivo en los años setenta tienen solo valor orientativo para prevenir actividades concretas y están enfocados a aportar información en la toma de decisiones. Ejemplos de este tipo de mapas son los analizados por Vrána (1984), que incluyen casos franceses, checoslovacos, polacos, rusos, búlgaros, alemanes y españoles (IGME, 1976). La siguiente generación de mapas, orientada a escalas más detalladas, representa el paso a la cartografía cuantitativa que integra información medible de variables, que permiten subdividir el territorio en recintos más 


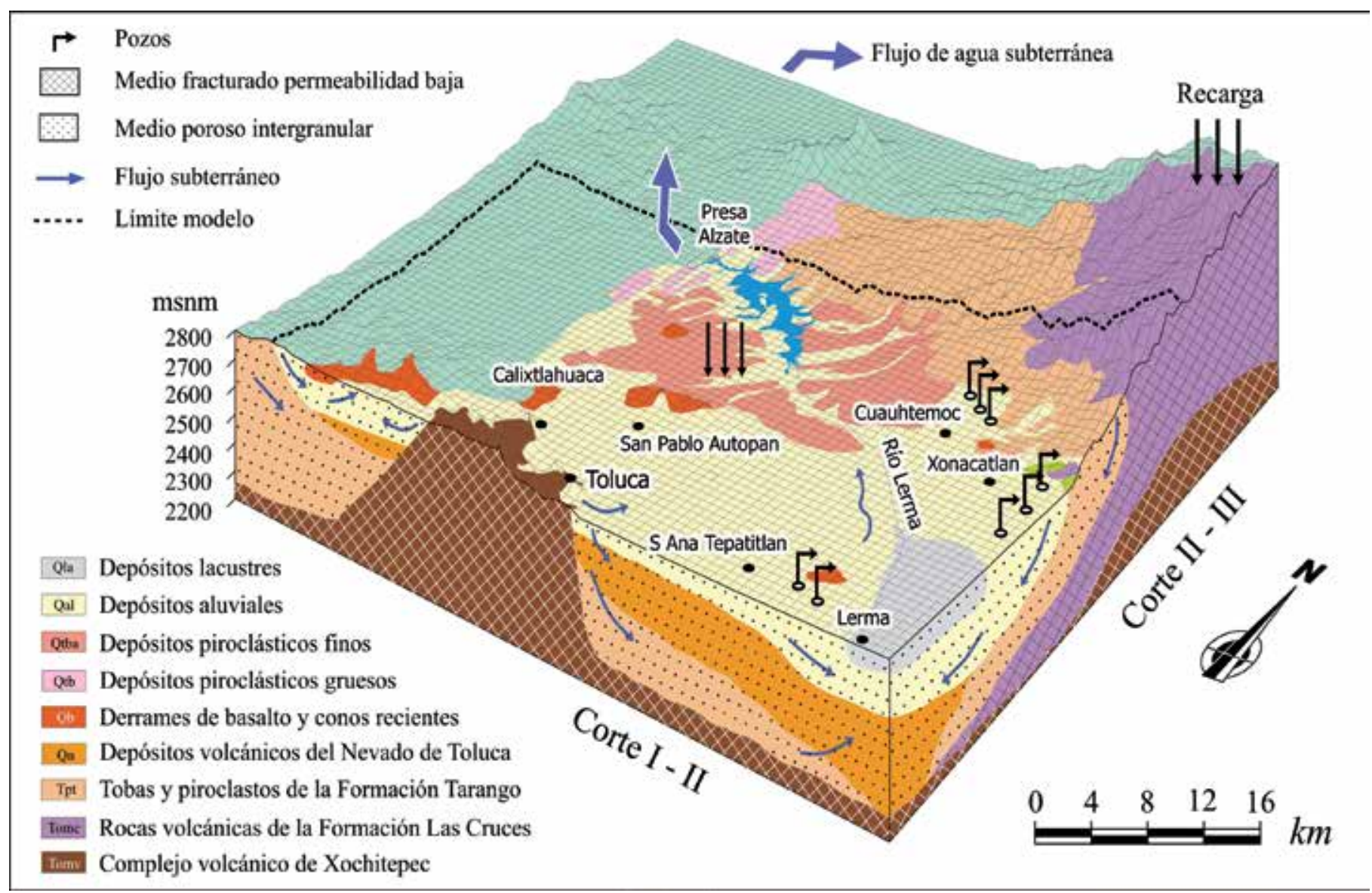

Figura 3. Esquema tridimensional del modelo conceptual del acuífero del valle deToluca y límites del modelo de flujo de agua subterránea. Figure 3. Three-dimensional conceptual scheme of the Toluca Valley aquifer and the boundary of the groundwater model.

pequeños de características más concretas (EVREN, 1998).

Con el concepto de vulnerabilidad ha ocurrido algo parecido; así, el concepto de vulnerabilidad a la contaminación de las aguas subterráneas introducido por Margat (1968) y utilizado por Albinet y Margat (1970), y posteriormente por Foster (1987), se basaba en la hipótesis de que el medio geológico y el suelo proporcionan un cierto grado de protección. Esta idea original evolucionó en el tiempo con la incorporación de nuevos elementos dando lugar a dos nuevas concepciones. De acuerdo a Brouyère et al. (2001), la vulnerabilidad intrínseca del agua subterránea a la contaminación, se fundamenta en las características geológicas, hidrológicas e hidrogeológicas inherentes con independencia de la naturaleza del contaminante. En cambio, la vulnerabilidad específica considera adicionalmente el comportamiento químico del contaminante y la vulnerabilidad del agua subterránea respecto a un contaminante en particular o grupo de contaminantes. En tal sentido, la estimación del riesgo, que tiene en cuenta la masa actual del contaminante, así como su concentración crítica, es un proceso jerárquico que se inicia con la vulnerabilidad intrínseca y se complementa con la vulnerabilidad específica (Brouyère et al., 2001; Frind et al., 2006).

Como elemento operacional en el desarrollo de los mapas de vulnerabilidad han tenido una gran importancia los sistemas de información geográfica (SIG), que permiten gestionar y analizar con suficiente rapidez y flexibilidad la información de tipo espacial muchas veces dispersa y almacenada en distintos formatos. Existe un gran número de programas disponibles en el mercado (Arc/Info, Maplnfo, Idrisi, ErMapper, llwis, entre otros), no obstante, para el presente trabajo se seleccionó el programa ArcView, ya que además de ser el más popular, es de tipo vectorial y fue diseñado específicamente para optimizar las representaciones de los proyectos realizados en Arc/Info, de suerte que con el tiempo se ha ido mejorando su estructura modular hasta convertirse en un software prácticamente independiente (ESRI, 1998). 
Gárfias, J., et al., 2017. Estimación de la vulnerabilidad del acuífero del valle de... Boletín Geológico y Minero, 128 (1): 25-42

\section{Análisis de la vulnerabilidad intrínseca de un acuífero}

Existen diversos métodos para la evaluación de la vulnerabilidad, cada uno de los cuales con sus propias limitaciones y exigencias en cuanto al tipo de datos adecuados a objetivos concretos, si bien entre los más valorados cabe destacar el God (Foster, 1987), Avi (Van Stempvoort et al., 1993), DRASTIC (Aller et al., 1987) y Sintacs (Civita, 1994). De todos ellos, en esta investigación se ha utilizado el método DRASTIC, ya que considera un mayor número de parámetros y su aplicación se encuentra estandarizada, tiene una mayor precisión y flexibilidad en estudios que requieren un detalle, así como por su facilidad de adaptarse a los sistemas de información geográfica (SIG).

En consecuencia, se trata del método más utilizado en Estados Unidos y Canadá, cuyas siglas corresponden a las iniciales de los parámetros considerados: profundidad al acuífero (Depth to water), recarga neta (net Recharge), litología del acuífero (Aquifer media), tipo de suelo (Soil media), topografía (Topography), litología de la zona no saturada (Impact of vadose zone) y conductividad hidráulica (hydraulic Conductivity). Cada parámetro tiene un factor de ponderación fijo que refleja su importancia en el cálculo de vulnerabilidad. Los parámetros pueden presentar valores de 1 a 10, en tanto que los factores o pesos varían de 1 a 5 , de modo que el índice de vulnerabilidad final ( $D i$ ) es la suma de los siete parámetros tal que:

$$
D_{i}=\sum_{J=1}^{7}\left(W_{j} \times R_{j}\right)
$$

donde $D_{i}$ es el índice de vulnerabilidad para cada unidad del mapa que varía entre 23 y 230 a medida que aumenta la vulnerabilidad, $W_{i}$ es el factor de peso para el parámetro $j$ y $R_{j}$ es el valor para el parámetro $j$ respectivamente. Los factores de pesos utilizados en el presente estudio han sido respectivamente de $5,4,3,2,1,5$ y 3 para los parámetros profundidad del nivel freático, recarga, litología del acuífero, tipo de suelo, topografía, litología de la zona no saturada y conductividad hidráulica. De acuerdo a Hamza et al. (2015), el método está basado en cuatro supuestos: (1) los contaminantes se depositan en la superficie del suelo, (2) los contaminantes se infiltran al subsuelo mediante el agua de recarga, (3) los contaminantes tienen la movilidad del agua que se infiltra y (4) es únicamente aplicable en zonas de superficie mayor o igual a $0.4 \mathrm{~km}^{2}$.

\section{Evaluación de la vulnerabilidad intrínseca en el acuí- fero del valle de Toluca}

Como se ha adelantado, la metodología DRASTIC es un sistema paramétrico de evaluación que incluye siete características a las que se asignan valores y un multiplicador, o peso, según su importancia. El resultado final es el Índice de Vulnerabilidad DRASTIC que, en realidad, se obtiene por superposición de polígonos de los siete parámetros, lo que permite encontrar un valor numérico para cada punto mediante la ecuación (1), en la que cada parámetro está afectado por un ponderador predeterminado y fijo, que refleja su importancia relativa en el cálculo de la vulnerabilidad (Hamza et al., 2015). La caracterización y obtención de los parámetros se efectuó como sigue:

- Profundidad al nivel freático. Se obtuvo a partir de los niveles estáticos medidos entre octubre y diciembre de 1997 (UNITECNIA, 1997), asignándose grados de vulnerabilidad crecientes para rangos de profundidad cada vez menores (Fig. 4). Determina el espesor de la zona no saturada que es la primera defensa natural del acuífero ante la contaminación, de modo que cuanto menor es esta distancia mayor es la probabilidad de que el contaminante alcance el agua subterránea.

- Recarga neta. Se ha estimado a partir de la información proporcionada por Ariel Consultores (Ariel Consultores, 1996), teniendo en cuenta las características de los materiales y la distribución de la precipitación media anual, de modo que a mayor recarga mayor susceptibilidad a la contaminación. En las áreas del fondo del valle, con una precipitación de 710 a $800 \mathrm{~mm}$ y una infiltración variable del $7,2 \%$ al $12,7 \%$, se estableció entre 51 y $102 \mathrm{~mm} / a n ̃ o$. Para las zonas montañosas se consideró la información obtenida por Salas (2012) y Calderhead et al. (2012a), quienes señalan valores variables para el coeficiente de infiltración de $4,5 \%$ a $26,6 \%$, dependiendo de la permeabilidad de los materiales, lo que asociado a una precipitación media de $1.000 \mathrm{~mm}$ permitió definir valores de recarga entre 45 y $270 \mathrm{~mm} / \mathrm{año}$.

- Litología del acuífero. Determina la velocidad de propagación de un contaminante y el tiempo disponible para que se desarrollen los procesos de atenuación; cuanto más permeable es la unidad geológica menor será la capacidad de atenuación del medio acuífero. Para su elaboración se partió de los trabajos de Ariel Consultores (1996) y Calderhead et al. (2011), teniendo en cuenta, desde un punto de vista cualitativo, el tipo de permeabilidad (Fig. 2), así como las características texturales de los diferentes materiales aflorantes, 


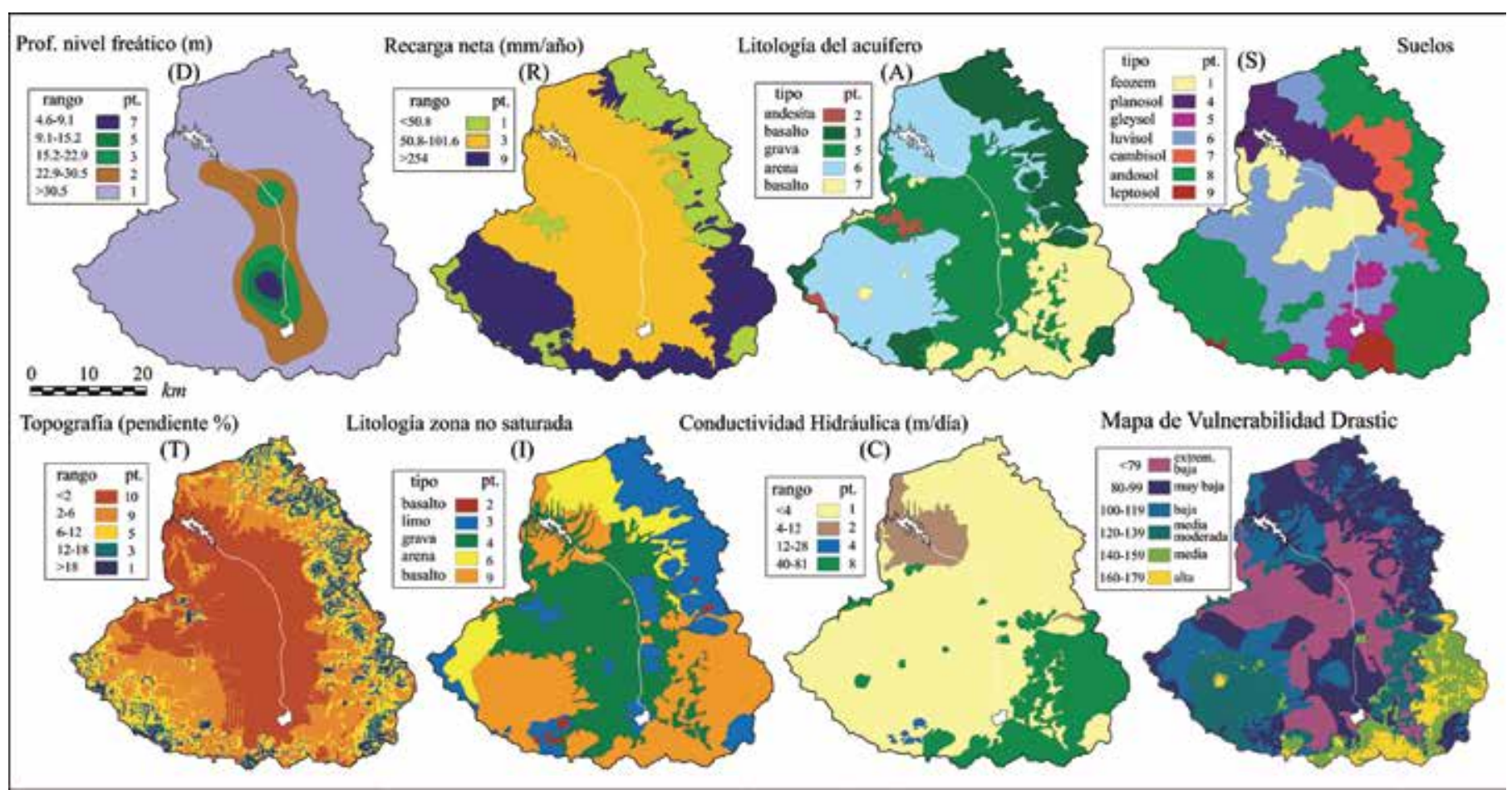

Figura 4. Distribución espacial de los factores del acuífero del Valle deToluca y mapa de vulnerabilidad de las aguas subterráneas generado en base a la metodología DRASTIC.

Figure 4. Spatial distribution of the Toluca valley aquifer factors and groundwater vulnerability map generated according to the DRASTIC method.

agrupándolas en cinco categorías con desigual capacidad para albergar agua, distinguiéndose principalmente materiales granulares (arenas $y$ grava) en la parte central y materiales rocosos en los bordes (basalto y andesita). En la Figura 4 se muestran los rangos y la distribución de puntuaciones DRASTIC asociadas a las distintas categorías litológicas existentes.

- Tipo de suelo. El suelo es la porción más superficial de la zona no saturada, y como resultado de su mayor contenido en arcilla, materia orgánica y de una extensa población bacteriana, la mayoría de los procesos que protegen a las aguas subterráneas son mucho más activos. De hecho, los suelos arcillosos (luvisol, planosol, vertisol), especialmente presentes hacia el fondo del valle de Toluca, son importantes en la función de atenuación sobre todo cuando la fuente de contaminación es difusa, como puede ser el caso de la aplicación de fertilizantes y pesticidas o deposiciones ácidas de carácter orgánico (Mendoza-Mejía y Orozco-Hernández, 2014). Sin embargo, hay que tener en cuenta que el suelo es muy vulnerable en sí mismo y que si la carga contaminante es aplicada por debajo del suelo, éste no contribuye en nada a reducir la vulnerabilidad del acuífero. En la Figura 4 se muestran las correspondientes puntuaciones asignadas y la distribución espacial de las unidades edáficas consideradas tomadas de la carta edafológica E142 escala 1:250.000 (INEGI, 1983).

- Topografía. Se estableció a partir del modelo digital de elevación de terreno E1402MDE a escala 1:250000 (INEGI, 1998). Dado su carácter matricial, el modelo se procesó inicialmente en el software IDRISI para generar las pendientes $y$, posteriormente, con el SIG para reclasificarlas de acuerdo a los rangos de la metodología DRASTIC. Finalmente se transfirió la información de formato raster a formato vectorial, obteniéndose el mapa de pendientes definitivo (Fig. 4).

- Litología de la zona no saturada. El tiempo que invierte el agua en alcanzar la zona saturada depende del espesor de la zona no saturada y del tipo de material atravesado que controla la duración de los procesos de atenuación de posibles contaminantes. Para su elaboración se tuvo en cuenta el mapa hidrogeológico (Fig. 2), a partir del cual se asignaron a cada litología la categoría que le corresponde de acuerdo con la metodología, así como la puntuación correspondiente (Fig. 4). 
Gárfias, J., et al., 2017. Estimación de la vulnerabilidad del acuífero del valle de... Boletín Geológico y Minero, 128 (1): 25-42

- Conductividad hidráulica. Para su cuantificación se tuvo en cuenta los valores derivados del proyecto de Ariel Consultores (1996) y Calderhead et al. (2011), información que se completó y verificó con medidas puntuales de la conductividad realizadas tanto in situ como en el laboratorio durante el desarrollo del presente estudio (Salas, 2012), obteniéndose la clasificación que se muestra en la Tabla 1 y su distribución espacial representada en la Fig. 4. Este factor determina la capacidad de los materiales para transmitir agua, controlando el flujo y el desplazamiento de posibles contaminantes, de suerte que cuanto mayor sea su valor mayor será la vulnerabilidad del acuífero.

Dado que el método DRASTIC permite asignar un valor numérico o índice de vulnerabilidad para cada punto del área estudiada facilitando la comparación de vulnerabilidades en distintas zonas, se procedió a la superposición de polígonos de cada uno de los parámetros descritos en dos fases con la ayuda del SIG. En la primera se determina la geometría de los nuevos polígonos generados por intersección de los existentes en los dos mapas que se superponen, asignando un identificador a cada nuevo polígono y reconstruyendo la topología resultante, en tanto que en la segunda se asignan los valores pertinentes a las variables temáticas asociadas al nuevo polígono. Esta operación se realizó mediante seis pasos sucesivos obteniéndose finalmente el mapa de la Figura 4, que corresponde a la zonificación de la vulnerabilidad del acuífero del valle de Toluca a la contaminación. En una primera instancia, dicho mapa proporciona una idea global de aquellas áreas del territorio que podrían verse más afectadas por vertidos ocasionales puntuales o por posibles focos contaminantes institucionalizados, como es el caso de los potenciales focos contaminantes representados por las zonas industriales y los vertederos de residuos sólidos (Figs. $6 a$ y $6 b)$.

En términos absolutos, las áreas de baja vulnerabilidad, con índice DRASTIC inferior a 119, representan el $68 \%$ del total de la superficie del área estudiada $\left(1.438 \mathrm{~km}^{2}\right)$, las de vulnerabilidad media, con índice comprendido entre 120 y 159 , suponen el $21 \%$ del total $y$, por último, las de alta vulnerabilidad, con índices superiores a 159, representan tan sólo el $11 \%$ del total (Tabla 2). No obstante, esta clasificación incorpora "índices cualitativos simples", que integran de forma simultánea características específicas del territorio que permiten controlar los procesos de transporte de solutos (Hamza et al., 2015). En tal sentido, es necesario aclarar que las zonas de más baja vulnerabilidad no indican que el acuífero subyacente no pueda ser contaminado, sino que la probabilidad de que se produzca una contaminación es menor.

La distribución espacial de la vulnerabilidad, tal y como se muestra en las Figuras 4 y $6 a$, refleja una alta a muy alta incidencia (11\%) principalmente en las zonas aledañas a los volcanes Tezontle y Zempoala, así como en algunas áreas del volcán Nevado de Toluca y en el sector suroriental de la Sierra de las Cruces. Las áreas de vulnerabilidad media $(9 \%)$ a media moderada $(12 \%)$ se sitúan en prolongación a la anterior, mostrando una mayor representación en el área del Nevado de Toluca y al Sureste de la Sierra de las Cruces. Así mismo, las clasificadas como de baja $(13 \%)$ a muy baja (33\%) vulnerabilidad, constituyen áreas intermedias entre los relieves periféricos y la parte más baja de la cuenca, que muestra una vulnerabilidad extremadamente baja (22\%), especialmente la localizada en el sector central del valle de Toluca. Resultados similares, aunque no coincidentes por la naturaleza del fenómeno, han sido reportados por

\begin{tabular}{|c|c|c|c|c|}
\hline Material & $\begin{array}{c}\boldsymbol{K} \\
(\boldsymbol{m} / \boldsymbol{s})\end{array}$ & $\begin{array}{c}\boldsymbol{S} \boldsymbol{s} \\
\left(\boldsymbol{m}^{-1}\right)\end{array}$ & $\begin{array}{c}\boldsymbol{S} \boldsymbol{y} \\
(\boldsymbol{\%})\end{array}$ & Capa \\
\hline Oal & $7 \times 10^{-5}$ & $4.7 \times 10^{-4}$ & 18 & 1 \\
Otba-Qb & $5 \times 10^{-5}$ & $5 \times 10^{-4}$ & 16 & $1-2$ \\
Otb-Tpt & $7 \times 10^{-6}$ & $2 \times 10^{-4}$ & 8 & $1-2$ \\
Tomc-Tpv-Tomv & $2 \times 10^{-7}$ & $7 \times 10^{-5}$ & 5 & $1-2$ \\
Otbn-On & $3 \times 10^{-6}$ & $8 \times 10^{-4}$ & 8 & $1-2$ \\
Oa & $2.3 \times 10^{-7}$ & $6 \times 10^{-4}$ & 13 & $1-2$ \\
Ola & $5 \times 10^{-6}$ & $7 \times 10^{-5}$ & 7 & 1 \\
\hline
\end{tabular}

Tabla 1. Valores representativos de la conductividad hidráulica, almacenamiento específico y rendimiento específico de las unidades hidrogeológicas del acuífero del valle de Toluca.

Table 1. Representative values of hydraulic conductivity, specific storage and specific yield for hydrogeologic units of the Toluca valley aquifer. 
Hamza et al. (2015), quienes resumieron estudios de vulnerabilidad desarrollados en 35 acuíferos, encontrándose que, por término medio, un $46 \%$ de las superficies totales analizadas se corresponden con la categoría de moderada a alta vulnerabilidad.

Un aspecto importante que no se contempla en la formulación de la metodología DRASTIC es la presencia de puntuales focos de contaminación. Bajo este contexto, hay que indicar la existencia en el área de estudio de un elevado número de vertederos, 80 de los cuales se localizan en zonas clasificadas por el método DRASTIC como de extremadamente baja a baja vulnerabilidad y 62 se sitúan en zonas de media-moderada a alta vulnerabilidad, en tanto que la mayor parte de las zonas industriales se sitúan en zonas de vulnerabilidad extremadamente baja y muy baja (Fig. 6a). En estas condiciones, pese a sus localizaciones, generalmente alejadas de las zonas urbanas, tanto los vertederos como los polígonos industriales constituyen, como se verá más adelante, importantes fuentes potenciales de contaminación.

\section{Vulnerabilidad de áreas específicas mediante la mo- delación numérica}

La vulnerabilidad intrínseca, tal y como se ha tratado, es una buena medida de la fragilidad de un acuífero, considerando como base de su análisis las características hidrogeológicas de la zona en estudio (Snyder et al., 1998). Sin embargo, este enfoque no tiene en cuenta la dinámica del sistema del flujo del agua subterránea, situación que constituye un factor fundamental para la estimación del riesgo. Así, un área de alta vulnerabilidad, por ejemplo, no se encuentra bajo riesgo de contaminación a menos que esté bajo la influencia de una fuente contaminante que le asigne un alto impacto de contaminación (Frind et al., 2006). Por tal motivo, y para aumentar el grado de confiabilidad obtenido en el estudio, el análisis se adecuó posteriormente a la actual situación de explotación del acuífero del valle de Toluca con la determinación simultánea de las zonas de captura, así como con la localización de las potenciales fuentes de contaminación. Dichas áreas, seleccionadas por razón de riesgo de contaminación, necesitan protección complementaria, ya que corresponden a explotaciones de agua subterránea destinadas al abastecimiento de agua potable e industrial (vulnerabilidad de pozos). En tal sentido, el enfoque propuesto pretende extender el concepto de vulnerabilidad intrínseca incluyendo el transporte advectivo de los contaminantes, sin entrar en la definición de las características químicas y concentraciones críticas del contaminante.
El transporte de contaminantes en el agua subterránea puede ser evaluado por medio de métodos analíticos, así como mediante modelos que simuIan el transporte advectivo o el transporte advectivo-dispersivo (Don et al., 2013; Gárfias et al., 2008b). Aunque los modelos de advección no pueden ser usados para estimar las concentraciones de soluto en el subsuelo, éstos representan un paso intermedio inapreciable entre los modelos de flujo de agua subterránea y los modelos advectivo-dispersivos más complejos (Frind et al., 2002). La importancia de la modelación numérica en la protección de las aguas subterráneas, mediante la simulación de flujo en tres dimensiones y la definición de las áreas de captura (advección), está bien documentada (Snyder et al., 1998; Frind et al., 2006; Don et al., 2013). Desde este punto de vista, en esta investigación se utilizó el modelo VisualModflow, que permite simular el flujo de agua subterránea en $3 \mathrm{D}$ en medios saturados, en conjunción con el modelo MODPATH, básicamente un programa de rastreo de partículas para identificar los tiempos de desplazamiento y las trayectorias de los contaminantes (Schlumberger Water Services, 2010). De acuerdo a Dong et al. (2013), modelos numéricos acoplados con un rastreo de partículas proporcionan un método más científico, por el hecho de considerar diferentes condiciones hidrogeológicas e intervalos de tiempo. En este sentido, en una primera fase del estudio, y a partir del mencionado programa, se adoptó como punto de partida el modelo numérico elaborado para la Comisión Nacional del Agua (Ariel Consultores, 1996), al que, posteriormente, se realizaron las oportunas adecuaciones al objeto de facilitar su empleo en la presente investigación.

Actualmente existen alrededor de 1000 pozos de explotación de agua subterránea en el valle de Toluca (Calderhead et al., 2012a), sin contabilizar las explotaciones clandestinas, que en número indeterminado explotan el acuífero, con el correspondiente efecto negativo sobre el almacenamiento del sistema acuífero. Partiendo de estos supuestos, se definió un total de cuatro escenarios que incluyen un cierto número de pozos representativos de las diferentes condiciones de explotación que permiten un análisis particularizado (Fig. 1). De este modo, para el primer escenario se seleccionaron parte de los pozos de abastecimiento del Sistema Lerma, lo que supone un total de 157 de los 236 existentes, cuyo volumen de extracción sirve para incrementar la disponibilidad de agua en la cuenca aledaña del valle de México. Un segundo escenario incluye 53 pozos de propiedad municipal-particular, cuyos volúmenes de explotación se destinan principalmente al área urbana de la ciudad de Toluca. Un tercer escenario 
Gárfias, J., et al., 2017. Estimación de la vulnerabilidad del acuífero del valle de... Boletín Geológico y Minero, 128 (1): 25-42

agrupa 13 pozos particulares de uso doméstico situados en la parte central y meridional del valle, que, por su importancia estratégica, constituyen fuentes que deben preservarse de los riesgos de contaminación. Por último, el cuarto escenario incluye 40 pozos particulares de uso industrial localizados en el centro del valle, al Noreste de la ciudad de Toluca, que por la actividad económica son de importancia estratégica, sin contar que se localizan en el área de mayor sobreexplotación y subsidencia del terreno. Además, se han incluido para el análisis la localización, ya comentada, de 142 vertederos de residuos sólidos y siete zonas industriales, que en su conjunto representan potenciales fuentes de contaminación (Figs. 6a y $6 \mathrm{c}$ ).

\section{Características principales del modelo de flujo de agua subterránea}

El modelo conceptual del área de estudio fue desarrollado a partir del modelo de elevación digital (MED), de las secciones estratigráficas de los pozos y de la información recolectada durante el trabajo de campo llevado a cabo en esta investigación. Los perfiles hidrogeológicos revelaron que el acuífero está compuesto principalmente de dos unidades hidrogeológicas, con intercalaciones de niveles de arcillas de espesor y geometría variables, que, en su conjunto, determinan un comportamiento hidrodinámico de carácter semiconfinado del sistema acuífero (Hancox et al., 2010; Calderhead et al., 2011). La Figura 3 muestra el esquema tridimensional del modelo conceptual del acuífero del valle de Toluca, así como parte de los límites noroccidentales del modelo de flujo de agua subterránea.

Con base a este esquema, el modelo original utiliza una malla de dos capas de 47 filas y 41 columnas, con 1927 celdas de $1 \mathrm{~km}$ de lado cada una (Figs. 5a y $5 b)$. Las celdas inactivas se situaron en el sector suroccidental (Nevado de Toluca) y nororiental (sierra de Las Cruces), en el primer caso debido a que su altura de más de $1000 \mathrm{~m}$ con respecto al valle propicia que la parte superior esté completamente drenada $y$, en el segundo, motivado por la naturaleza impermeable de la formación Las Cruces. Igualmente hacia el centro del valle se sitúa un reducido número de celdas inactivas correspondientes a las andesitas de la formación Xochitepec. Para fines de esta investigación, se procedió a una discretización espacial complementaria refinando la malla a un arreglo de 94 filas y 82 columnas, con 7708 celdas de $500 \mathrm{~m}$ de lado. El refinamiento se realizó particularmente por la presencia de áreas de interés, como es el caso de la existencia de zonas de discontinuidad del acuífero y zonas donde existen una gran concentración de pozos, situación que contribuyó a una convergencia aceptable de la calibración, tanto en lo que se refiere a la calibración en estado estacionario como a la calibración transitoria.

\section{Propiedades hidráulicas regionales}

Se consideraron siete valores de conductividad, $K, y$ otros tantos para el coeficiente de almacenamiento, $S s$, y el rendimiento específico, $S y$, de acuerdo con la naturaleza de las formaciones geológicas (Tabla 1). Esta información fue extraída de los resultados de las pruebas de bombeo que se desarrollaron en distintas épocas (Ariel Consultores, 1996; Caldehead et al., 2011), cuyo fin principal fue determinar las propiedades hidráulicas de las unidades hidrogeológicas del acuífero. Con el objeto de definir la configuración de los niveles piezométricos, que, a su vez, condicionan la dirección del flujo de agua subterránea, se consideró la distribución del nivel estático con respecto al nivel del mar para los años 1970, 1984 y 1996, en el entendido que para estos periodos las series de datos son las más completas (Ariel Consultores, 1996; Calderhead et al., 2011).

\section{Condiciones de frontera e impulsiones del sistema acuífero}

Las condiciones de frontera fueron definidas como de carga general, considerándose el flujo horizontal proveniente de la infiltración generada en las sierras de Las Cruces y La Iglesia, a una cota de $2800 \mathrm{msnm}$ y conductancia de 20 y $30 \mathrm{~m}^{2} /$ día en las capas 1 y 2 respectivamente (Fig. 5). El flujo procedente del Nevado de Toluca, con un área de recarga remota a $3000 \mathrm{msnm}$ y conductancia de $20 \mathrm{~m}^{2} /$ día, se le aplicó a la capa 2, en tanto que a la capa 1 se le asignó una conductancia de $5 \mathrm{~m}^{2} /$ día e idéntica carga remota. Así mismo, se consideró una frontera tipo dren, correspondiente a la salida por flujo horizontal hacia el valle de Ixtlahuaca, en un tramo de cauce donde el río Lerma funciona en condiciones efluentes a una cota de $2530 \mathrm{msnm}$ y una conductancia de $150 \mathrm{~m}^{2} /$ día (Fig. 5). La condición inicial se fijó con la información disponible de los niveles estáticos correspondientes al año 1970.

Por otra parte, teniendo en cuenta el valor de la precipitación media anual, estimada en $770 \mathrm{~mm}$, se consideró una recarga vertical de $55 \mathrm{~mm} / a n ̃ o$, que representa el $7.1 \%$ del volumen total de la precipitación (Ariel Consultores, 1996). Además, dado que en 
la época de estudio se dieron en la región extensas superficies de afloramientos con niveles piezométricos someros, se consideró una condición de frontera de evapotraspiración correspondiente a una lámina

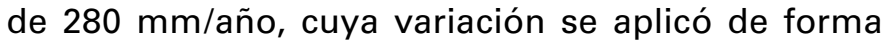
lineal hasta los $10 \mathrm{~m}$ de profundidad del nivel de agua subterránea (Schlumberger Water Services, 2010). En cuanto a las extracciones por bombeo, se consideraron los datos del censo de aprovechamientos de 1996, cuya información consigna un total de 530 pozos con un volumen de extracción de $327 \mathrm{Mm}^{3} /$ año. Los datos disponibles para 1970 y 1984 son incompletos e inciertos; así, por ejemplo, el volumen de extracción con destino al uso doméstico para 1970 está claramente sobreestimado si lo comparamos con el censo de aprovechamientos de 1984, que bajo análogas condiciones es casi cinco veces menor (Ariel Consultores, 1996).

\section{Calibración del modelo}

La calibración del modelo se efectúo en dos etapas secuenciales: en una primera instancia, se abordó la modelación del flujo de agua subterránea en régimen estacionario, al objeto de reproducir el sistema de flujo en condiciones básicas e iníciales, y, posteriormente, una vez calibrados los diferentes parámetros hidráulicos que intervienen en el proceso, pasar a simular en condiciones transitorias. Para este fin, se consideraron como condición inicial los niveles estáticos del agua subterránea de 1970, cuyos valores obtenidos en 55 multipiezómetros permitieron la estimación de las cargas hidráulicas independientes de la variación temporal de las impulsiones. Esta etapa de la calibración permitió el ajuste de la distribución de la conductividad hidráulica y las condiciones de frontera en el sistema acuífero.

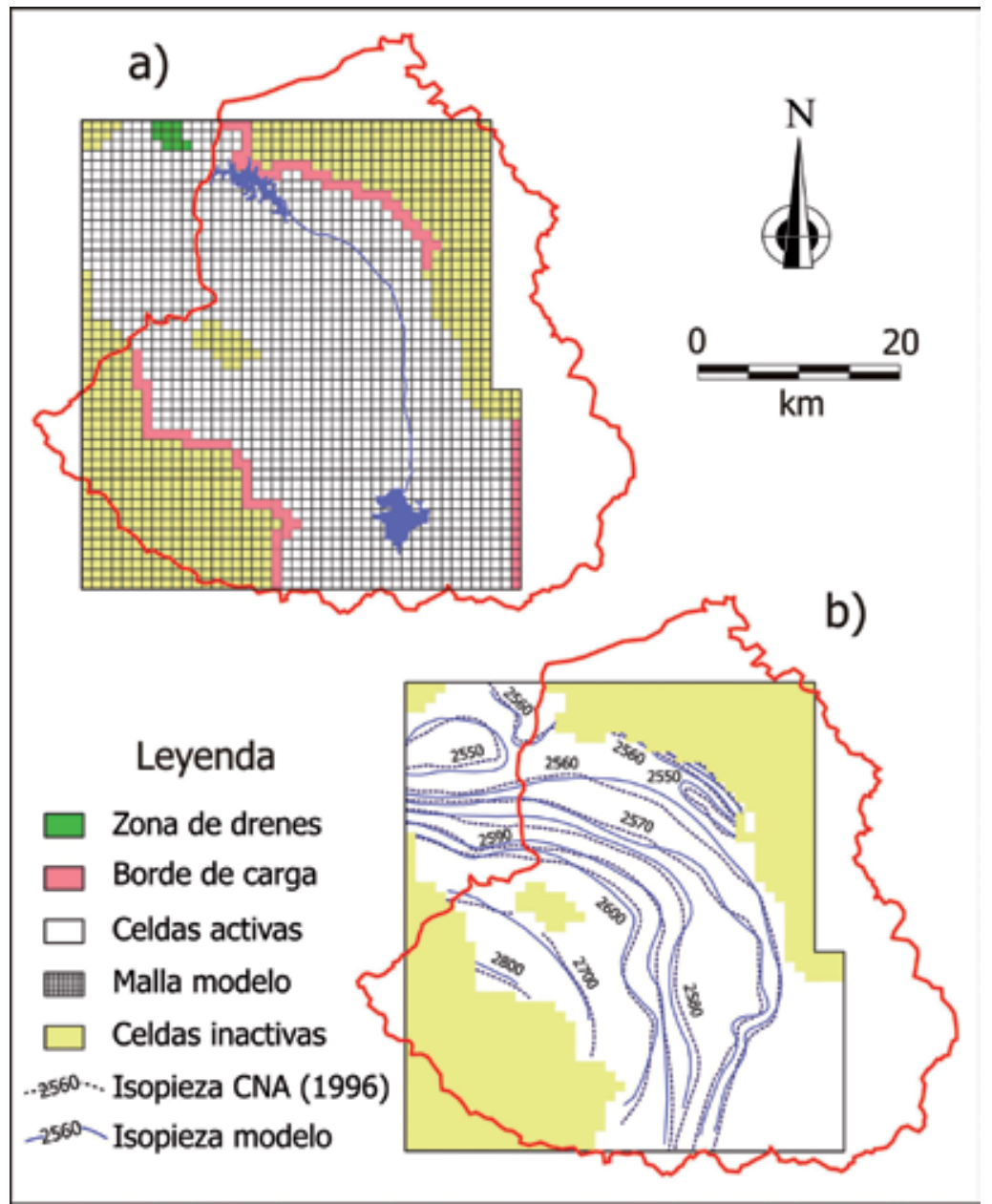

Figura 5. (a) Discretización del área de estudio y condiciones de frontera del modelo de flujo de agua subterránea y (b) comparación de niveles estáticos del agua subterránea simulados y observados en la calibración en estado estacionario para el año 1996.

Figure 5. (a) Discretisation of the study area and boundary conditions of the groundwater model and (b) comparison of simulated and observed groundwater levels under steady-state calibration for 1996. 
Gárfias, J., et al., 2017. Estimación de la vulnerabilidad del acuífero del valle de... Boletín Geológico y Minero, 128 (1): 25-42

En base a los valores obtenidos, se procedió a la calibración en estado transitorio considerando dos periodos de esfuerzo, un primer periodo de 1970 a 1984 y un segundo periodo de 1985 a 1996. Los valores de los coeficientes de almacenamiento fueron los principales valores ajustados durante la calibración transitoria, cuyos resultados fueron evaluados por comparación con las variaciones temporales de las cargas hidráulicas simuladas y las observadas en los multipiezómetros. Al observar las configuraciones que se muestran en la Figura $5 b$ se aprecia, en general, una buena coincidencia, con algunas diferencias que se pueden calificar como aceptables dada la estructura de las unidades hidrogeológicas y su comportamiento hidrodinámico. Por último, para simular el rastreo de partículas hacia atrás y los tiempos de transito (TOT), se asignaron los resultados obtenidos de la simulación efectuada con los datos de 1996 a los valores iniciales de la calibración en estado estacionario, cuyo objetivo final era establecer el campo de velocidades que permitiera delinear las zonas de captura para un tiempo de transito en particular.

\section{Mapa de vulnerabilidad adecuado mediante el rastro de partículas advectivas}

Con el modelo calibrado, el siguiente paso consistió en delimitar el desplazamiento advectivo de partículas contaminantes en un tiempo de tránsito de 50 años (18250 días), suficiente para que se atenúen los contaminantes más persistentes (Frind et al., 2006). Para ello se utilizó el programa MODPATH (módulo del Visual Modflow), que permite delinear la trayectoria de dichas partículas tanto hacia adelante (en el sentido del flujo) como hacia atrás (áreas de captura). El procedimiento se inició situando en la capa superior del modelo partículas hipotéticas contaminantes alrededor de cada uno de los pozos de abastecimiento. A continuación, con el modelo MODPATH se estableció la trayectoria de las partículas hacia atrás (pathlines) y, a partir de ellas, se delimitaron las áreas de protección específicas consideradas como de alta vulnerabilidad, ya que cualquier contaminación que en ellas se pudiera generar y alcanzara el acuífero podría deteriorar la calidad de las aguas subterráneas de los pozos objeto de la protección. Finalmente, las áreas así definidas se importaron al SIG para superponerlas a la cartografía de vulnerabilidad generada con el método DRASTIC (Fig. 6c).

La metodología descrita se aplicó a los cuatro escenarios seleccionados (Fig. 1), cuyos resultados de manera conjunta se muestran en las Figuras $6 b$ y 6c. La superposición final de las áreas obtenidas ha permitido generar un nuevo mapa de vulnerabilidad orientado a la protección específica de puntos sensibles de la cuenca, al que se ha denominado mapa adecuado de vulnerabilidad del acuífero del valle de Toluca (Fig. 6c). Así mismo, en la Figura 6b se muestra una imagen panorámica del valle de Toluca donde se ilustra la trayectoria de las partículas advectivas correspondientes a posibles puntos susceptibles de contaminación para áreas de captura de 50 años, así como la localización de las zonas industriales. Con el objeto de sintetizar los resultados, la Figura $6 \mathrm{c}$ muestra en dos dimensiones, además de los elementos de la Figura $6 \mathrm{~b}$, la localización de los vertederos de residuos sólidos, que en conjunto suponen el principal riesgo de la contaminación de las aguas del acuífero del valle de Toluca.

\section{Análisis y discusión de resultados}

Los resultados obtenidos se resumen en dos mapas de vulnerabilidad, uno elaborado a partir del método DRASTIC (Figs. 4 y $6 a$ ) y otro resultado de su combinación con la modelación numérica (Figs. $6 \mathrm{~b}$ y $6 \mathrm{c}$ ). Al primero de ellos, el hecho de haber sido desarrollo sobre un SIG de tipo vectorial (ArcView) y no matricial le confiere a la cartografía una serie de ventajas, permitiendo conocer las propiedades del medio y de los parámetros que contribuyen a obtener un índice de vulnerabilidad determinado. EI mapa DRASTIC muestra cómo el área del fondo del valle y las serranías nororientales se caracterizan por índices de baja a extremadamente baja vulnerabilidad. En el primer caso debido a la baja tasa de recarga y, en el segundo, debido al carácter prácticamente impermeable de las rocas aflorantes.

Las zonas de vulnerabilidad media se localizan al Sureste, en las inmediaciones de la sierra de La Iglesia, así como en las faldas del Nevado de Toluca. En ambos sectores, los parámetros más influyentes son las litologías de la zona no saturada y del acuífero, si bien en el sector suroccidental la recarga es una característica importante. Las zonas de vulnerabilidad alta se sitúan en las elevaciones de la sierra de La Iglesia, coincidiendo con áreas de alta recarga sobre afloramientos de rocas fracturadas y alta permeabilidad. Una característica particular de esta configuración, dada su relación con los focos potenciales de contaminación, lo constituye la ubicación de los vertederos de residuos sólidos donde 62 de los 142 vertederos contabilizados se encuentran en el radio de acción de vulnerabilidad catalogada como de media-moderada a alta. En cambio, las zonas industriales, a pesar de representar un riesgo claro 


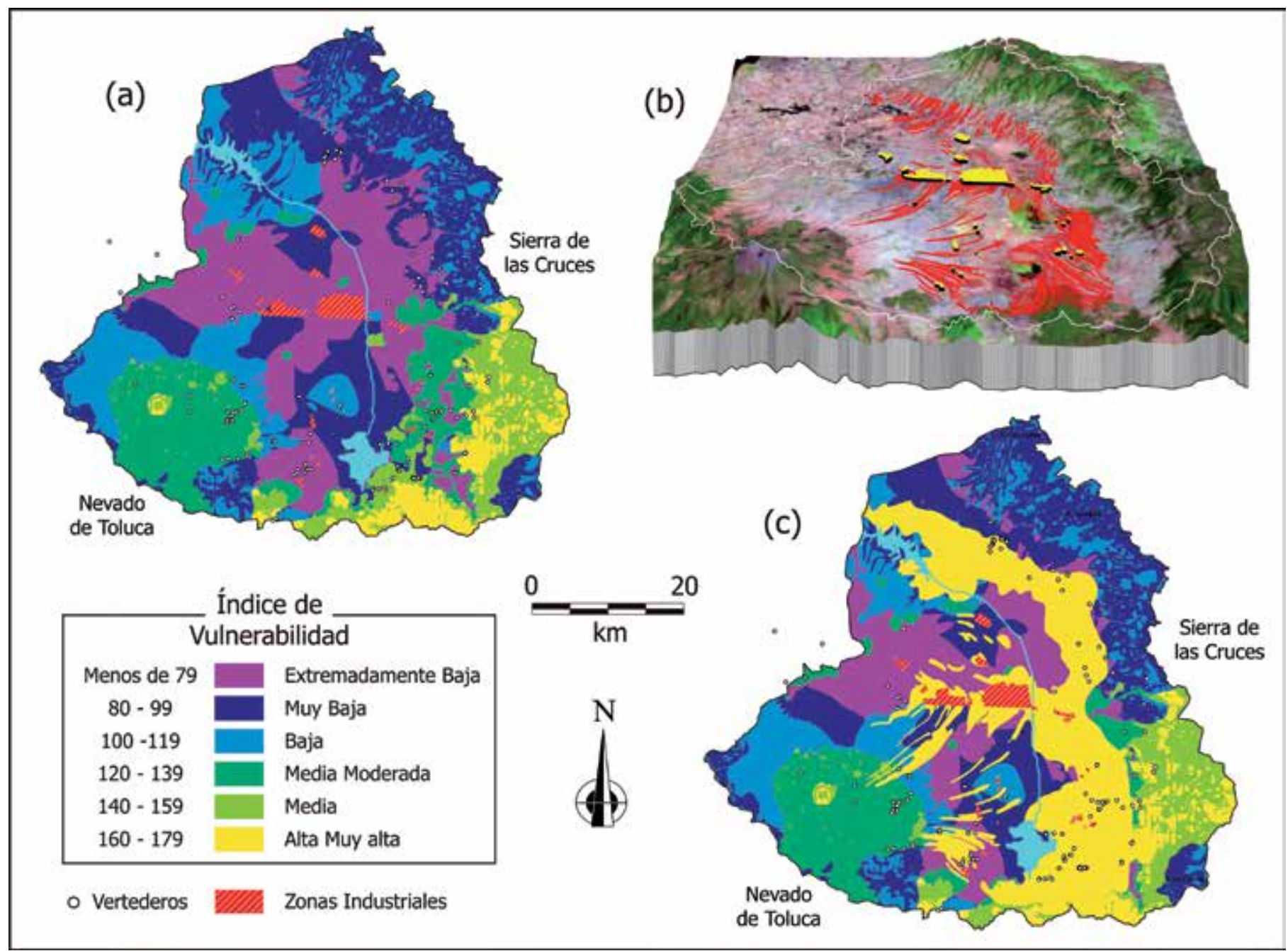

Figura 6. (a) Mapa de vulnerabilidad generado de acuerdo a la metodología DRASTIC, (b)Trayectoría de partículas de los campos de pozos para áreas de captura de 50 años (4 escenarios) y (c) Mapa combinado de vulnerabilidad de aguas subterráneas derivado de los enfoques DRASTIC y modelación numérica, incluyendo las zonas industriales y los vertederos de residuos sólidos.

Figure 6. (a) Groundwater vulnerability map generated according to the DRASTIC method, (b) Particle tracking flow paths from the weII-fields for 50 years of the capture areas (4 scenarios) and (c) combined groundwater vulnerability map derived from the DRASTIC and numerical modelling approaches, including industrial zones and landfills sites.

de contaminación potencial, no parecen constituir a priori un grave peligro porque se localizan en zonas de vulnerabilidad extremadamente baja y muy baja, salvo un reducido número de zonas industriales que se localizan en áreas de media a moderada vulnerabilidad.

Partiendo del hecho de que los resultados obtenidos por el método DRASTIC no son absolutos, se procedió a su modificación mediante la delineación de los tiempos de tránsito de partículas advectivas, obteniendo áreas de protección complementaria que fueron consideradas de alta y muy alta vulnerabilidad. Esta conversión se basa en el hecho de que cualquier contaminante introducido o vertido accidentalmente desde estas áreas tiene la posibilidad de afectar el agua de los pozos de abastecimiento, por lo que resulta necesario evitar que se realice cualquier actividad potencialmente contaminante sin las debidas medidas de protección. Así, existen diferencias significativas en cuanto al tamaño de las áreas a proteger, como puede reconocerse en las figuras que dieron lugar al mapa de vulnerabilidad modificado (Figs. 6a y $6 c$ ). Ello se debe a que las trayectorias de las partículas tienen una mayor presencia en aquellos puntos donde la velocidad del flujo subterráneo es mayor, como sucede en el extremo suroriental y central de la zona modelada coincidiendo con una alta concentración de pozos, situación que ha generado fuertes 
Gárfias, J., et al., 2017. Estimación de la vulnerabilidad del acuífero del valle de... Boletín Geológico y Minero, 128 (1): $25-42$

abatimientos del nivel freático, que al mismo tiempo concentra la mayor cantidad de zonas industriales.

La cartografía de vulnerabilidad modificada muestra una serie de diferencias con respecto a la generada por el método DRASTIC (Figs. 6). Estas diferencias se sintetizan en la Tabla 2 en la que se aprecian los cambios sufridos entre los índices de vulnerabilidad de ambos mapas, observándose que los mayores cambios se producen en las zonas de alta vulnerabilidad. Ello es debido a que las áreas delimitadas para su protección complementaria, mediante la técnica del rastreo de partículas, se consideraron, como ya se ha indicado, de alta y muy alta vulnerabilidad al objeto de limitar al máximo las actividades potencialmente contaminantes que en ellas podrían generarse, motivo por el que los porcentajes de las áreas de baja y media vulnerabilidad disminuyeron significativamente, de $68 \%$ a $50 \%$ y de $21 \%$ a $16 \%$, en tanto que las áreas de alta a muy alta vulnerabilidad se modifican de $11 \%$ a $34 \%$ (Tabla 2 ).

De este modo, la adecuación del mapa de vulnerabilidad conlleva a que 108 de los 142 vertederos existentes se localicen ahora en áreas de media-moderada a alta vulnerabilidad, situación que implica un incremento del $74 \%$. Situación similar ocurre con las zonas industriales, aunque de manera más dramática dado que ahora la práctica totalidad de las mismas se localizan en áreas catalogadas como de alta a muy alta vulnerabilidad, con excepción de algunas que se encuentran en sectores de vulnerabilidad extremadamente baja a baja. En este contexto, cobra una relevancia crítica la zona industrial ubicada en la porción central del valle, que dada su importancia estratégica y económica constituye el área donde se producen los mayores niveles de abatimiento y efectos de subsidencia del terreno por la sobreexplotación del agua subterránea.

\section{Conclusiones}

El objetivo principal de la presente investigación fue evaluar el grado de vulnerabilidad del acuífero del vaIle de Toluca, perteneciente a la cuenca del río Lerma en el Estado de México, mediante la aplicación combinada de la metodología DRASTIC y el rastreo advectivo de partículas, lo que ha permitido generar sendos mapas de vulnerabilidad complementarios de utilidad para la gestión de los recursos hídricos subterráneos y el ordenamiento territorial del área estudiada. En consecuencia, con el objeto de proteger la explotación del agua subterránea de los pozos de producción, es esencial desarrollar un buen conocimiento del sistema de flujo y una adecuada delineación de las áreas aledañas a los campos de pozos donde las fuentes potenciales de contaminación deberían ser controladas de manera continua en el futuro.

El uso combinado de los conceptos de vulnerabilidad intrínseca y transporte advectivo permitió crear la base conceptual para la adecuación del mapa de vulnerabilidad generado a partir de la metodología DRASTIC. En efecto, la aplicación de un modelo de flujo con rastreo de partículas ha permitido delimitar zonas más vulnerables que requieren una especial protección. En estos sectores cualquier actividad potencialmente contaminante podría provocar, a la postre, el deterioro de la calidad del agua subterránea. Dichas áreas, consideradas como de alta vulnerabilidad, se solaparon a la cartografía original DRASTIC, obteniendo un nuevo mapa en el que quedó incorporada la protección complementaria de las áreas de captura de los pozos para tiempos de tránsito de 50 años.

El nuevo mapa muestra una serie de mejoras con respecto al generado por el método DRASTIC, ante la

\begin{tabular}{|l|c|c|c|c|c|c|c|}
\hline \multirow{2}{*}{ Vulnerabilidad } & \multirow{2}{*}{$\begin{array}{c}\text { Índice de } \\
\text { Vulnerabilidad }\end{array}$} & \multicolumn{2}{|c|}{ DRASTIC } & \multicolumn{3}{c|}{ Modelo } \\
\cline { 2 - 8 } & extremadamente baja $<79$ & 460 & 22 & & 409 & 19 & \\
\hline \multirow{3}{*}{ Baja } & muy baja 80-89 & 708 & 33 & 68 & 443 & 21 & 50 \\
& baja 100-119 & 270 & 13 & & 200 & 10 & \\
\hline \multirow{2}{*}{ Media } & media-moderada 120-139 & 253 & 12 & 21 & 218 & 10 & 16 \\
\hline Alta & media 140-159 & 201 & 9 & & 124 & 6 & 16 \\
\hline
\end{tabular}

Tabla 2. Comparación de los resultados de la vulnerabilidad de las aguas subterráneas derivadas de los métodos DRASTIC y modelación numérica.

Table 2. Comparison of groundwater vulnerability results derived from the DRASTIC and numerical modelling approach. 
imposibilidad de este último de considerar el sistema de flujo del acuífero, ya que los índices DRASTIC son únicamente calculados para una determinada posición y no reflejan las condiciones hidrodinámicas del flujo del agua subterránea. Los cambios que se obtienen tras la adecuación afectan a los diferentes índices, cuya tasa ponderada de cambio puede estimarse en un $23 \%$, valor que puede considerarse como el grado de incertidumbre de la vulnerabilidad intrínseca respecto al mapa de vulnerabilidad adecuado. Los vertederos de residuos sólidos, a pesar de estar alejados de las zonas urbanas, representan ahora un riesgo sensible por su localización ya que el $76 \%$ se sitúan en zonas de alta vulnerabilidad, constituyendo especial peligro aquellos que se encuentran próximos a los pozos del sistema Lerma. Así mismo, por su ubicación, las zonas industriales son igualmente de especial interés como potenciales fuentes contaminantes dado que un $84 \%$ de sus superficies se asientan sobre terrenos catalogados como de alta a muy alta vulnerabilidad frente a un $16 \%$ de vulnerabilidad baja a extremadamente baja. A este respecto las zonas con una mayor incidencia son sin duda las que se encuentran en la parte central del valle de Toluca, donde además tiene lugar una mayor sobreexplotación que se asocia a descensos diferenciales del subsuelo.

En resumen, se han confeccionado dos mapas de vulnerabilidad de gran importancia, ya que además de servir de pauta para la elaboración de políticas para la protección del acuífero del valle de Toluca, su correcta utilización permitiría alcanzar el necesario uso sostenible de los recursos hídricos subterráneos que en la actualidad presentan problemas de sobreexplotación. Así mismo, podrían constituir una herramienta imprescindible para la gestión del territorio $y$, por lo tanto, podrían conducir a evaluaciones de la vulnerabilidad más concluyentes sobre el uso del territorio y a la toma de medidas de protección contra la contaminación.

\section{Agradecimientos}

Los autores agradecen al Consejo Nacional de Ciencia y Tecnología (CONACyT, 201800), al Consejo Mexiquense de Ciencia y Tecnología (COMECyT) y a la Universidad Autónoma del Estado de México (UAEM, 3640, 3641, 3642) por el apoyo financiero para el desarrollo de la presente investigación, así como a la Comisión Nacional del Agua (CONAGUA) por el apoyo financiero, la información de base suministrada y la colaboración en los trabajos de campo (Convenio CGPEAYS-UAEM- 07/2013).

\section{Referencias}

Alaniz-Álvarez, S.A. y Nieto-Samaniego, A.F. 2005. El sistema de fallas Taxco-San Miguel de Allende y la Faja Volcánica Trans-Mexicana, dos fronteras tectónicas del centro de México activas durante el Cenozoico. Boletín de la Sociedad Geológica Mexicana, Volumen Conmemorativo del Centenario, LVII (1), 65-82.

Aller, L., Ennet, T., Leher, J.H., Petty, R.J. y Hackett, G. 1987. DRASTIC: a standardized system for evaluating ground water pollution potential using hydrogeological settings. EPA/600/2-87-036, $455 \mathrm{pp}$.

Ariel Consultores. 1996. Estudio de Simulación Hidrodinámica y Diseño Óptimo de las Redes de Observación de los acuíferos de Calera, San Luis Potosí y Toluca. (Tomo 3: Acuífero de Toluca). Ariel Consultores, S.A., México, D.F., 308 p.

ESRI. 1998. Introduction to ArcView GIS. Environmental Systems Research Institute, Educational Services.

EVREN. 1998. Vulnerabilidad a la contaminación de las aguas subterráneas por actividades urbanísticas en la Comunidad Valenciana. Evaluación de Recursos Naturales S.A., Pub. de Divulgación Técnica, Colección Cartografía Temática, Valencia, España, 58 pp.

Brouyère, S., Jeannin, P.Y., Dassargues, A., Golscheider, N., Popescu, I.C., Sauter, M., Vadillo, I. y Zwahlen, F., 2001, Evaluation and validation of vulnerability concepts using a physically based approach, Proc. of the 7th Conf. on Limestone Hydrology and Fissured Media, J. Mudry \& F. Zwahlen (Eds.), Sciences et Techniques de l'Environnement, Université de Franche-Comté, Mémoire $\mathrm{n}^{\circ} 13$, pp. 67-72.

Calderhead, A., Martel, R., Gárfias, J., A. Rivera, A. y Therrien, R. 2012b. Sustainable Management for Minimizing Land Subsidence of an Over-Pumped Volcanic Aquifer System: Tools for Policy Design. Water Resources Management, 26, 7, 1847-1864.

Calderhead, A., Martel, R., Gárfias, J., Rivera, A. y Therrien, R. 2012a. Pumping dry: an increasing groundwater budget deficit induced by urbanization, industrialization, and climate change in an over-exploited volcanic aquifer. Environmental Earth Sciences Journal, 66, 7, 1753-1767.

Calderhead, A., Therrien, R., Rivera, A., Martel, R. y Gárfias, J. 2011. Simulating pumping-induced regional land subsidence in a complex aquifer system. Advances in Water Resources, 34(1), pp. 83-97.

Civita, M. 1994. Le carte della vulnerabilita` degli acquiferi all'inquinamento. Teoria \& practica (Aquifer vulnerability maps to pollution) (in Italian). Pitagora Ed, Bologna, $325 \mathrm{p}$.

CONAGUA. 2014. Atlas del Agua en México. Secretaría del Medio Ambiente y Recursos Naturales, 142 p.

Don., Y., Xu, H. y Guomin, L. 2013. Wellhead protection area delineation using multiple methods: a case study in Beijing. Environemtal Earth Sciences, 70, 481-488.

Expósito, J.L., Esteller, M.V., Paredes, J., Rico, C. y Franco, R. 2010. Groundwater Protection Using Vulnerability Maps and Wellhead Protection Area (WHPA): A Case 
Gárfias, J., et al., 2017. Estimación de la vulnerabilidad del acuífero del valle de... Boletín Geológico y Minero, 128 (1): 25-42

Study in Mexico. Water Resources Management, 2010, 24, 15, 4219-4236.

Ferrari, L., Orozco-Esquivel, T., Manea, V. y Manea, M. 2012. The dynamic history of the Trans-Mexican Volcanic Belt and the Mexico subduction zone. Tectonophysics, Vol. 522-523, pp. 122-149.

Foster, S. 1987. Fundamental concepts in aquifer vulnerability, pollution risk and protection strategy. Vulnerability of soil and groundwater to pollutants. En: Van Duijvenbooden, W. and Van Waegenigh H.G. (ed). TNO Committee on Hydrological Research Information \# 38, The Hague, 69-86.

Frind, E.O., Molson, J.W. y Rudolph, D.L. 2006. Well vulnerability: a quantitative approach for source water protection. Ground Water 44:732-742.

Frind, E.O., Muhammad, D.S. y Molson, J.W. 2002. Delineation of threedimensional well capture zones for complex multi-aquifer systems. Ground Water 40(6):586-598.

García-Palomo, A., Macías, J.L. y Garduño, V.H. 2000. Miocene to Recent structural evolution of the Nevado de Toluca Volcano region, central Mexico. Tectonophysics, Special Volume, Post-Laramide magmatism and tectonics in Mexico plate interaction, 318, 281-302.

García-Palomo, A., Macías, J.L., Arce, J.L., Capra, L., Garduño, V.H. y Espíndola, J.M. 2002. Geology of Nevado de Toluca Volcano and surrounding areas, central Mexico. Bolulder, Colorado, Geological Society of America Map and Chart Series MCH089, 26 p.

García-Palomo, A., Zamorano, J.J., López-Miguel, C., Galván-García, A., Carlos-Valerio, V., Ortega, R. y Macías, J.L. 2008. El arreglo morfoestructural de la Sierra de las Cruces, México central. Revista Mexicana de Ciencias Geológicas, 25 (1), 158-178.

Gárfias, J., Llanos, H. y L. Bibiano, L. 2008a. Uso Racional y Sostenible de los recursos hídricos del acuífero del valle de Toluca. Revista Ciencia Ergo Sum, 15-1, 61-72.

Gárfias, J., Expósito, J.L. y Llanos, H. 2008b. Delimitación de las zonas de protección mediante métodos analíticos y un modelo numérico de agua subterránea, Acuífero Margarita, Cuba. Boletín Geológico y Minero, 119 (1), 7-20.

Hamza, S.M., Ahsan, A., Imteaz, M.A., Rahman, A., Mohammad, T.A. y Ghazali, A.H. 2015. Accomplishment and subjectivity of GIS-based DRASTIC groundwater vulnerability assessment method: a review. Environmental Earth Sciences; 73(4), 3063-3076.

Hancox, J., Gárfias, J., Aravena, R. y Rudolph, D.L. 2010. Assessing the Vulnerability of over-exploited volcanic aquifer systems using Multiparameter Analysis, Toluca Basin, Mexico. Environmental Earth Sciences; 59(8), 1643-1660.
IGME. 1976. Mapa de vulnerabilidad a la contaminación de los mantos acuíferos de la España Peninsular, Baleares y Canarias. Primer esquema cualitativo. $2^{a}$ ed., mem. explic., mapa esc. 1:1000000. Serv. Public. Min. Industria, Madrid.

INEGI. 1983. Carta Edafológica E14-2 escala 1:250000. Instituto Nacional de Estadística Geografía e Informática. Gobierno Edo. de México, Toluca.

INEGI. 1998. Modelo Digital de Elevación E1402MDE escala 1:250000. Instituto Nacional de Estadística Geografía e Informática. Gobierno Edo. de México, Toluca.

Rudolph, D.L., R. Sultan, R., Gárfias, J. y McLaren, R.G. 2006. Significance of Enhanced Infiltration due to Groundwater Extraction on the Disappearance of a Large Wetland System: Toluca Basin, Mexico. Hydrogeology Journal, 14 (1-2), 115-130.

Salas, J. 2012. Determinación espacial de la recarga mediante el diseño e instalación de instrumentación en pozos de monitoreo y simulación de la infiltración en la zona vadosa. Centro Interamericano de Recursos del Agua (CIRA), Universidad Autónoma del Estado de México, Toluca, México, 186 p.

Schlumberger Water Services, 2010. Visual MODFLOW, Modular three-dimensional finite-difference groundwater flow model, version 2010.1. Schlumberger Water Services, Waterloo, Ontario, $676 \mathrm{p}$.

Snyder, D.T., Wilkinson, J.M. y Orzol, L.L. 1998. Use of a ground-water flow model with particle tracking to evaluate ground-water vulnerability, Clark Country, Washington. USGS Water-Supply Paper 2488, 63 pp.

UAEM. 1993. Problemática Ambiental de los Recursos Hídricos en la Cuenca Alta del Río Lerma. Seminario Ambiental sobre el Ambiente, 1, 170-181.

UNITECNIA 1997. Actualización de Mediciones Piezométricas de los acuíferos Reactivados en los vaIles de Toluca y Atlacomulco-Ixtlahuca, en el Estado de México. México, DF.: Unitecnia, 26 p.

Van Stempvoort, D., Evert, L. y Wassenaar, L. 1993. Aquifer vulnerability index: a GIS compatible method for groundwater vulnerability mapping. Can. Wat. Res. Jour., 18, 25-37.

Vrána, M. 1984. Methodology for construction of groundwater protection maps. En: Kozlovsky E.A. (ed). Hydrogeological Principles of Groundwater Protection, Moscow, Unesco/Unep, 1, 147-149.

Martín del Campo, M.A., Esteller, M.V., Exposito, J.L. y Hirata, R. 2014. Impacts of urbanization on groundwater hydrodynamics and hydrochemistry of the Toluca Valley aquifer (Mexico). Environmental Monitoring and Assessment, 186(5), 2979-2999.

Mendoza-Mejía, J.B. y Orozco-Hernández, M.E. 2014. Análisis de la vulnerabilidad biofísica a los riesgos por inundación en la zona metropolitana de Toluca, México. Revista Luna Azul, 38, 86-104.

Recibido: junio 2015

Revisado: noviembre 2015

Aceptado: febrero 2016

Publicado: marzo 2017 